\title{
Combining Ability Studies in Quality Protein Maize (Zea mays L.)
}

\author{
K. Sukumar*, V. Hemalatha, V. Narsimha Reddy, \\ S. Narender Reddy and D. Srinivasa Chary
}

Professor Jayashankar Telangana State Agricultural University, Telangana, India

*Corresponding author

\begin{tabular}{l} 
Ke y w o r d s \\
$\begin{array}{l}\text { Quality protein } \\
\text { maize, Combining } \\
\text { ability, General } \\
\text { combining ability }\end{array}$ \\
Article Info \\
$\begin{array}{l}\text { Accepted: } \\
\text { 15 June } 2020 \\
\text { Available Online: } \\
\text { 10 July } 2020\end{array}$ \\
\hline
\end{tabular}

\section{A B S T R A C T}

Combining ability studies were conducted in 9X 9 half diallel fashion in quality protein maize (QPM) at Agricultural Research Station, Ramnagar, Adilabad during Kharif, 2015 to determine GCA and SCA effects of parents and single crosses respectively. The overall study indicated that the parents, QPM-39, QPM-45, QPM 10057-2, QPM-10-1 and QPM-15 can be considered as good general combiners and genetically worthy parents, as they contributed favourable genes for grain yield and its components at all the three locations and in pooled analysis. Combining ability analysis revealed that the parents, QPM 58, QPM-45, QPM-3-3, QPM-39 and QPM-15 were found to be good general combiners for earliness. The parent, QPM-39 and QPM-45 were good general combiners for grain yield, days to 50 per cent anthesis, days to 50 per cent silking, days to maturity (all towards earliness), ear girth, ear length, number of kernels per ear, number of kernels per row, 100-kernel weight, shelling percentage. In addition, QPM-45 was a good combiner for tryptophan and lysine contents.

\section{Introduction}

Maize (Zea mays L.) is the third most important cereal crop of India after rice and wheat. It is a member of grass family Poaceae, tribe Maydeae and is highly cross pollinated crop. It has assumed greater significance due to its demand for food, feed and industrial utilization. Nearly $49 \%$ of the maize produced is being utilized as raw material in the poultry feed industry. Maize being a $\mathrm{C} 4$ plant and fertilizer responsive has very high yielding ability coupled with higher amount of cross pollination. Hence, offers tremendous scope for the plant breeders for genetic improvement.

It is considered as queen of cereals due to its high genetic potentiality and adaptability. It is delight for the plant breeders to work on this 
important crop due to the presence of high genetic variability and adaptability. Several people consume maize as staple food to meet their protein and calories requirement.

Malnutritional problems were observed where maize was the staple food especially in young children, pregnant and lactating mothers in tribal communities. High proportion of zein (seed storage protein of maize) fraction which is completely devoid of lysine and tryptophan is the primary cause of poor protein quality in maize because of which its biological value and net protein utilization was less.

However, maize breeders strived hard to improve the nutritional aspects in maize by incorporating modified opaque 2 into normal maize so that the digestability and biological values were improved with the enhanced levels of lysine and tryptophan contents to combat problems of malnutrition. QPM has balanced leucine : isoleucine ratio with the enhanced niacin levels helps in preventing pellagra. It is a better alternative to animal protein which is costly and plays a major role in meeting infant and lactating mother food requirements.

Combining ability is of special importance in cross-pollinated crops like maize as it helps in identifying potential in breds in order to produce the hybrids and synthetics. Combining ability is the relative ability of a genotype to transmit its desirable performance to its progenies. Selection of parents, information on heterotic patterns and combining ability among maize genotypes is desirable in hybrid development. In addition, it provides an insight into the genetic architecture of various characters that enables the breeder to design effective breeding plan. The study involving quality protein maize focused to assess the gene action for quantitative and qualitative traits and to explore heterotic hybrid combinations.
General combining ability (GCA) is attributed to additive gene effects and additive $\mathrm{x}$ additive epistasis and is theoretically fixable. On the other hand, specific combining ability attributable to non-additive gene action may be due to dominance or epistasis or both and is non-fixable.

\section{Materials and Methods}

The selected parents (9) were crossed in half diallel fashion to develop single cross hybrids at Agricultural Research Station (ARS), Adilabad during Rabi, 2014-15 and the hybrids thus developed were during kharif, 2015.The diallel set of 36 crosses along with 9 parents and two checks viz., DHM-117 and Vivek QPM 9 were sown in Randomized Block Design replicated thrice. Each entry was sown in two rows of four meters length with a spacing of $75 \mathrm{~cm}$ between rows and 20 $\mathrm{cm}$ between the plants. The recommended fertilizers of $\mathrm{N}, \mathrm{P}$ and $\mathrm{K}$ were applied in the ratio of 120: 80: $60 \mathrm{~kg} \mathrm{ha}^{-1}$. The entire $\mathrm{P}$ and $\mathrm{K}$ and half dose of nitrogen was applied as basal, while remaining half dose of $\mathrm{N}$ in two equal split doses at knee height and tasseling stages.

Weeding operations, necessary plant protection measures were taken up to protect the crop from pests and diseases as per the recommendations along with the timely irrigation schedules to raise a healthy crop.

Data was recorded on the important characters viz., days to 50 per cent anthesis, days to 50 per cent silking, Anthesis silking interval(ASI), days to maturity, plant height $(\mathrm{cm})$, ear height $(\mathrm{cm})$, ear length $(\mathrm{cm})$, ear girth $(\mathrm{cm})$, number of kernel rows per ear, number of kernels per row, grain yield (g/plant),100 kernel weight (g), shelling percentage, crude protein (\%), lysine content $(\%)$ and tryptophan content $(\%)$. 


\section{Results and Discussion}

The pooled analysis of variance for combining ability revealed significant differences in all the locations for all the characters revealed that the parental lines exhibited highly significant differences among themselves for all the characters studied indicating the greater diversity existed in the lines. The crosses also exhibited significant differences, indicating varying performance of cross-combinations. The parents vs. crosses also exhibited significant for all the traits except for anthesis silking interval thus considerable amount of average heterosis was reflected in hybrids. The interaction of parents with environment is significant for ear height while, interaction of hybrids with environment was significant for ear length. However, the interaction of parent $v s$. hybrids with environment revealed significant differences for all the traits except for plant height, anthesis silking interval and 100-kernel weight. These results revealed the importance of combining ability studies for indicating the variability present in the material studied and providing the information on scope for identifying promising parents and hybrid combinations and improving yield through its attributing traits.

Significant negative sca effects for days to 50 per cent anthesis were recorded in 25 hybrids. The hybrids, QPM-10-1 X QPM-77, QPM53-2-2 X QPM-77, QPM10057-2 X QPM-532-2 and QPM-15 X QPM-77 were good specific combiners for days to 50 per cent anthesis for achieving earliness (Table 2).These results are comparable with findings of Jagadish Kumar et al., (2010) and Kumar et al., (2012) who reported the non-additive gene action for days to 50 per cent tasseling.

Among the parents, QPM-3-3, QPM-45 and QPM-39 recorded significant negative gca effects for days to 50 per cent silking and identified as best general combiner combiners for earliness that can further be exploited in the development of early duration hybrids. These results are comparable with findings of Bhavana et al., (2011) and Sumalini (2012) who reported the additive gene action for days to 50 per cent silking. Among the 36 hybrids, 24 hybrids recorded negatively significant $s c a$ effects. The hybrids, QPM-10-1 X QPM-77, QPM-53-2-2 X QPM-77,QPM-53-2-2 X QPM-15 and QPM-15 X QPM-77 which recorded the highest significant negative $s c a$ effects at the three locations and in pooled analysis indicating that they were good specific combiners for earliness. These results are comparable with findings of Singh et al., (2010) and who reported the non- additive gene action for days to 50 per cent silking. However, none of the parents showed significant negative effects for anthesis silking interval.

Among the parents, QPM58, QPM-45, QPM15, QPM3-3 and QPM-39 recorded significantly negative gca effects and they were good general combiners for earliness. These results are comparable with findings of Murthy et al., (1981) and Sanghi et al., (1982) who reported additive gene action for days to maturity.

As many as 20 hybrids recorded significantly negative $s c a$ effects. The promising for days to maturity were QPM-53-2-2 X QPM-77, QPM-53-2-2 X QPM-10-1 and QPM-10-X QPM-45 which expressed the highest significant negative effects indicating that they are early duration hybrids which are highly essential in the present scenario of climate change with uncertain rain fall pattern, shrinking of water resources and depletion of ground water table. These results are in agreement with the findings of Ram Reddy et al., (2012) who reported the non additive gene action for days to maturity. 
Table.1 Pooled analysis of variance for combining ability (diallel) for yield and yield components in Quality protein maize.

\begin{tabular}{|c|c|c|c|c|c|c|c|c|c|c|}
\hline & d.f & $\begin{array}{l}\text { Plant } \\
\text { height } \\
(\mathbf{c m})\end{array}$ & $\begin{array}{c}\text { Days to } \\
50 \% \\
\text { anthesis }\end{array}$ & $\begin{array}{c}\text { Days to } \\
50 \% \\
\text { Silking }\end{array}$ & $\begin{array}{c}\text { Anthesis } \\
\text { silking } \\
\text { interval }\end{array}$ & $\begin{array}{c}\text { Ear } \\
\text { height } \\
(\mathbf{c m})\end{array}$ & $\begin{array}{c}\text { Ear } \\
\text { length } \\
(\mathrm{cm})\end{array}$ & $\begin{array}{l}\text { Ear girth } \\
\quad(\mathrm{cm})\end{array}$ & $\begin{array}{c}\text { Number } \\
\text { of kernel } \\
\text { rows per } \\
\text { ear }\end{array}$ & $\begin{array}{c}\text { Number } \\
\text { of } \\
\text { kernels } \\
\text { per row }\end{array}$ \\
\hline Environments & 2.00 & $\begin{array}{c}2367.78 \\
* *\end{array}$ & $348.87 * *$ & $412.55 * *$ & $3.68 * *$ & $\begin{array}{c}1157.10 \\
* *\end{array}$ & $305.33 * *$ & $180.66 * *$ & $\begin{array}{c}102.91 \\
* *\end{array}$ & $97.77 * *$ \\
\hline $\begin{array}{l}\text { Blocks within } \\
\text { Environments }\end{array}$ & 6.00 & 61.01 & 0.32 & 0.14 & 0.17 & 0.97 & 0.10 & 0.04 & 0.21 & 0.11 \\
\hline Treatments & 44.00 & $\begin{array}{c}2754.78 \\
* *\end{array}$ & $174.96 * *$ & $174.27 * *$ & $\begin{array}{c}0.39 \\
* *\end{array}$ & $\begin{array}{c}1367.62 \\
* *\end{array}$ & $274.59 * *$ & $37.70 * *$ & $20.73 * *$ & $\begin{array}{l}406.15 \\
* *\end{array}$ \\
\hline Parents & 8.00 & $\begin{array}{c}3909.50 \\
* *\end{array}$ & $122.03 * *$ & $125.14 * *$ & $\begin{array}{c}0.81 \\
* *\end{array}$ & $893.75 * *$ & $25.77 * *$ & $25.19 * *$ & $22.81 * *$ & $\begin{array}{l}202.28 \\
* *\end{array}$ \\
\hline Hybrids & 35.00 & $\begin{array}{c}2470.82 \\
* *\end{array}$ & $99.49 * *$ & $97.01 * *$ & $\begin{array}{c}0.31 \\
*\end{array}$ & $\begin{array}{c}1514.03 \\
* *\end{array}$ & $252.46 * *$ & $31.83 * *$ & $20.62 * *$ & $\begin{array}{l}453.98 \\
* *\end{array}$ \\
\hline $\begin{array}{l}\text { Parents vs. } \\
\text { Hybrids }\end{array}$ & 1.00 & $\begin{array}{c}3455.53 \\
* *\end{array}$ & $\begin{array}{c}3239.93 \\
* *\end{array}$ & $\begin{array}{c}3271.11 \\
* *\end{array}$ & 0.07 & $34.09 * *$ & $\begin{array}{c}3039.89 \\
* *\end{array}$ & $343.02 * *$ & $7.95 * *$ & $\begin{array}{l}362.85 \\
* *\end{array}$ \\
\hline $\begin{array}{c}\text { Treatment } \mathbf{x} \\
\text { Environments }\end{array}$ & 88.00 & 3.23 & $2.00 * *$ & $2.40 * *$ & 0.17 & 1.28 & $0.99 * *$ & 0.16 & 0.28 & 1.33 \\
\hline $\begin{array}{c}\text { Parent } \\
\text { xEnvironments }\end{array}$ & 16.00 & 0.95 & 0.30 & 0.43 & 0.16 & $3.67 *$ & 0.01 & 0.01 & 0.09 & 0.00 \\
\hline $\begin{array}{c}\text { Hybrids x } \\
\text { Environments }\end{array}$ & 70.00 & 3.36 & 0.00 & 0.16 & 0.17 & 0.07 & $0.98 * *$ & 0.01 & 0.03 & 1.10 \\
\hline $\begin{array}{c}\text { Parent vs. } \\
\text { Hybrids x Env. }\end{array}$ & 2.00 & 17.16 & $85.39 * *$ & $96.34 * *$ & 0.36 & $24.29 * *$ & $8.96 * *$ & $6.65 * *$ & $10.59 * *$ & $19.81 * *$ \\
\hline Error & 264.00 & 32.67 & 0.29 & 0.44 & 0.20 & 2.15 & 0.34 & 0.13 & 0.30 & 1.82 \\
\hline Total & 404.00 & 334.71 & 21.41 & 21.83 & 0.23 & 156.37 & 31.85 & 5.12 & 3.03 & 46.20 \\
\hline
\end{tabular}


Table.1 Contd...

\begin{tabular}{|c|c|c|c|c|c|c|c|c|}
\hline & d.f & $\begin{array}{l}\text { Days to } \\
\text { Maturity }\end{array}$ & $\begin{array}{l}100 \text { Kernel } \\
\text { Weight }(g)\end{array}$ & $\begin{array}{c}\text { Shelling } \\
\text { percentage }\end{array}$ & $\begin{array}{c}\text { Crude } \\
\text { Protein } \\
\text { content }(\%)\end{array}$ & $\begin{array}{l}\text { Tryptophan } \\
\text { content }(\%)\end{array}$ & $\begin{array}{c}\text { Lysine } \\
\text { content } \\
(\%)\end{array}$ & $\begin{array}{c}\text { Grain Yield } \\
\text { ( g/plant ) }\end{array}$ \\
\hline Environments & 2.00 & $833.65 * *$ & $84.39 * *$ & $190.21 * *$ & $5.81 * *$ & $0.07 * *$ & $0.14 * *$ & $723.32 * *$ \\
\hline $\begin{array}{l}\text { Blocks within } \\
\text { Environments }\end{array}$ & 6.00 & 0.59 & 1.16 & 2.04 & 0.08 & 0.00 & 0.01 & 2.62 \\
\hline Treatemnts & 44.00 & $87.95 * *$ & $118.86 * *$ & $309.16 * *$ & $6.87 * *$ & $0.03 * *$ & $0.35 * *$ & $2991.29 * *$ \\
\hline Parents & 8.00 & $199.36 * *$ & $126.50 * *$ & $75.24 * *$ & $11.82 * *$ & $0.02 * *$ & $0.25 * *$ & $402.39 * *$ \\
\hline Hybrids & 35.00 & $50.19 * *$ & $112.01 * *$ & $122.32 * *$ & $2.98 * *$ & $0.02 * *$ & $0.26 * *$ & $1539.34 * *$ \\
\hline $\begin{array}{c}\text { Parents } \\
\text { vs.Hybrids }\end{array}$ & 1.00 & $518.32 * *$ & $297.67 * *$ & $8719.95 * *$ & $103.63 * *$ & $0.46 * *$ & $4.21 * *$ & $74520.61 * *$ \\
\hline $\begin{array}{c}\text { Treatment * } \\
\text { Environments }\end{array}$ & 88.00 & $3.07 * *$ & 0.21 & 2.23 & 0.01 & $0.00 * *$ & 0.00 & $8.80 * *$ \\
\hline $\begin{array}{c}\text { Parent * } \\
\text { Environments }\end{array}$ & 16.00 & 1.22 & 0.08 & 0.05 & 0.00 & 0.00 & 0.00 & 0.00 \\
\hline $\begin{array}{c}\text { Hybrids * } \\
\text { Environments }\end{array}$ & 70.00 & 0.27 & 0.20 & 1.59 & 0.00 & 0.00 & 0.00 & $10.85 * *$ \\
\hline $\begin{array}{l}\text { Parent vs.Hybrids } \\
\text { * Env. }\end{array}$ & 2.00 & $115.95 * *$ & 1.52 & $42.16 * *$ & $0.45 * *$ & $0.03 * *$ & $0.07 * *$ & 7.38 \\
\hline Error & 264.00 & 1.45 & 0.88 & 2.40 & 0.08 & 0.00 & 0.00 & 3.00 \\
\hline Total & 404.00 & 15.33 & 14.00 & 36.70 & 0.83 & 0.00 & 0.04 & 333.28 \\
\hline
\end{tabular}

* Significant at $5 \%$ level; ** Significant at $1 \%$ level 
Table.2 Estimates of general combining ability effects for parents

\begin{tabular}{|c|c|c|c|c|c|c|c|c|c|c|c|c|c|c|c|c|}
\hline & $\begin{array}{c}\text { Plant } \\
\text { height } \\
\text { ( cm ) }\end{array}$ & $\begin{array}{c}\text { Days to } \\
50 \% \\
\text { anthesis }\end{array}$ & $\begin{array}{c}\text { Days to } \\
50 \% \\
\text { Silking }\end{array}$ & $\begin{array}{l}\text { Anthesis } \\
\text { silking } \\
\text { interval }\end{array}$ & $\begin{array}{c}\text { Ear } \\
\text { height } \\
(\mathbf{c m})\end{array}$ & $\begin{array}{c}\text { Ear } \\
\text { length } \\
(\mathbf{c m})\end{array}$ & $\begin{array}{l}\text { Ear } \\
\text { girth } \\
(\mathbf{c m})\end{array}$ & $\begin{array}{c}\text { Number } \\
\text { of kernel } \\
\text { rows per } \\
\text { ear }\end{array}$ & $\begin{array}{c}\begin{array}{c}\text { Number } \\
\text { of }\end{array} \\
\text { kernels } \\
\text { per row }\end{array}$ & $\begin{array}{l}\text { Days to } \\
\text { Maturity }\end{array}$ & $\begin{array}{c}100 \\
\text { Kernel } \\
\text { Weight } \\
\text { (g) }\end{array}$ & $\begin{array}{c}\text { Shelling } \\
\text { percentage }\end{array}$ & $\begin{array}{c}\text { Crude } \\
\text { Protein } \\
\text { content } \\
(\%)\end{array}$ & $\begin{array}{l}\text { Tryptophan } \\
\text { content (\%) }\end{array}$ & $\begin{array}{c}\text { Lysine } \\
\text { content } \\
(\%)\end{array}$ & $\begin{array}{c}\text { Grain } \\
\text { Yield } \\
\text { ( g/plant ) }\end{array}$ \\
\hline \multicolumn{17}{|c|}{ Parents } \\
\hline QPM58 & $-3.79 * *$ & $-0.33 * *$ & $-0.24 *$ & 0.09 & $-2.50 * *$ & $1.02 * *$ & $-0.31 * *$ & $-0.40 * *$ & $0.98^{* *}$ & $-0.70 * *$ & $-2.16^{* *}$ & $-0.62 * *$ & $0.20 * *$ & -0.01 & $-0.06 * *$ & $-0.12 * *$ \\
\hline QPM10057-2 & $4.78 * *$ & $0.25^{* *}$ & $0.34 * *$ & 0.09 & $4.26^{* *}$ & $1.28 * *$ & $0.28 * *$ & $0.48 * *$ & $2.39 * *$ & -0.10 & $0.92 * *$ & $1.20 * *$ & $0.15^{* *}$ & $0.02 * *$ & $0.03 * *$ & $0.14 * *$ \\
\hline QPM-53-2-2 & -0.19 & $0.37 * *$ & $0.31 * *$ & -0.06 & $-1.26^{* *}$ & $-0.21 * *$ & $-0.61^{* *}$ & $-0.53 * *$ & $-2.82 * *$ & -0.13 & $-0.64 * *$ & $-1.20 * *$ & $0.24 * *$ & 0.00 & $-0.03 *$ & $-0.32 * *$ \\
\hline QPM-10-1 & 1.48 & $1.89^{* *}$ & $1.79 * *$ & -0.09 & $1.16^{* *}$ & $-0.18 * *$ & -0.04 & $-0.25 * *$ & -0.11 & $0.78^{* *}$ & 0.13 & 0.29 & $-0.27 * *$ & 0.00 & 0.01 & $0.14 * *$ \\
\hline QPM-45 & $3.27 * *$ & $-0.96 * *$ & $-1.08 * *$ & -0.12 & $3.16^{* *}$ & $0.38^{* *}$ & $0.62 * *$ & $0.26^{* *}$ & $0.63^{* *}$ & $-1.37 * *$ & $0.42 * *$ & $0.98 * *$ & $-0.30 * *$ & $0.01^{*}$ & $0.04 * *$ & $0.39 * *$ \\
\hline QPM-15 & $-5.79 * *$ & $0.43^{* *}$ & $0.52 * *$ & 0.09 & $-2.65^{* *}$ & $-0.96 * *$ & -0.06 & $0.28 * *$ & -0.09 & $-0.67 * *$ & $1.33 * *$ & $-1.14 * *$ & $0.19 * *$ & $-0.02 * *$ & $0.05 * *$ & $0.18 * *$ \\
\hline QPM3-3 & $3.60 * *$ & $-3.14 * *$ & $-3.14 * *$ & 0.00 & $0.83^{* *}$ & $0.80 * *$ & $0.26^{* *}$ & $0.37 * *$ & $-1.00 * *$ & $-0.98 * *$ & $0.52 * *$ & $-0.80 * *$ & 0.05 & -0.01 & -0.01 & $-0.09 * *$ \\
\hline QPM-39 & 0.54 & $-0.69 * *$ & $-0.69 * *$ & 0.00 & $-1.84 * *$ & $0.94 * *$ & $0.84 * *$ & $0.49 * *$ & $2.67 * *$ & $-1.22 * *$ & $2.62 * *$ & $1.98 * *$ & 0.04 & $-0.01 * *$ & -0.02 & $0.78 * *$ \\
\hline QPM-77 & $-3.89 * *$ & $2.19 * *$ & $2.19 * *$ & 0.00 & $-1.17 * *$ & $-3.08 * *$ & $-0.98 * *$ & $-0.70 * *$ & $-2.65 * *$ & $4.39 * *$ & $-3.15^{* *}$ & $-0.68 * *$ & $-0.30 * *$ & $0.02 * *$ & -0.02 & $-0.96^{* *}$ \\
\hline SE (gi) & 1.002 & 0.088 & 0.107 & 0.075 & 0.234 & 0.066 & 0.051 & 0.066 & 0.200 & 0.113 & 0.160 & 0.179 & 0.041 & 0.003 & 0.011 & 0.27 \\
\hline SE (gi-gj) & 1.503 & 0.132 & 0.160 & 0.113 & 0.351 & 0.099 & 0.076 & 0.099 & 0.300 & 0.169 & 0.240 & 0.269 & 0.061 & 0.005 & 0.016 & 0.41 \\
\hline
\end{tabular}


Table.3 Estimates of Specific combining ability effects for Single crosses

\begin{tabular}{|c|c|c|c|c|c|c|c|c|c|c|c|c|c|c|c|c|}
\hline & $\begin{array}{l}\text { Plant } \\
\text { height } \\
(\text { cm ) }\end{array}$ & $\begin{array}{c}\text { Days to } \\
50 \% \\
\text { anthesis }\end{array}$ & $\begin{array}{l}\text { Days to } \\
50 \% \\
\text { Silking }\end{array}$ & $\begin{array}{l}\text { Anthesis } \\
\text { silking } \\
\text { interval }\end{array}$ & $\begin{array}{c}\text { Ear } \\
\text { height } \\
(\mathbf{c m})\end{array}$ & $\begin{array}{c}\text { Ear } \\
\text { length } \\
(\mathbf{c m})\end{array}$ & $\begin{array}{l}\text { Ear } \\
\text { girth } \\
(\mathbf{c m})\end{array}$ & $\begin{array}{c}\text { Number } \\
\text { of kernel } \\
\text { rows per } \\
\text { ear }\end{array}$ & $\begin{array}{c}\text { Number } \\
\text { of kernels } \\
\text { per row }\end{array}$ & $\begin{array}{c}\text { Days to } \\
\text { Maturity }\end{array}$ & $\begin{array}{c}100 \\
\text { Kernel } \\
\text { Weight } \\
\text { (g) }\end{array}$ & $\begin{array}{c}\text { Shelling } \\
\text { percentage }\end{array}$ & $\begin{array}{c}\text { Crude } \\
\text { Protein } \\
\text { content } \\
(\%)\end{array}$ & $\begin{array}{l}\text { Tryptophan } \\
\text { content }(\%)\end{array}$ & $\begin{array}{c}\text { Lysine } \\
\text { content } \\
(\%)\end{array}$ & $\begin{array}{c}\text { Grain Yield } \\
\text { (g/plant ) }\end{array}$ \\
\hline \multicolumn{17}{|l|}{ Crosses } \\
\hline $\begin{array}{c}\text { QPM58 X } \\
\text { QPM10057-2 }\end{array}$ & $8.94 * *$ & -0.32 & -0.36 & -0.05 & $-12.01 * *$ & $-0.61 * *$ & $-1.30^{* *}$ & 0.24 & -0.09 & $-1.74 * *$ & $-1.97 * *$ & $-3.16^{* *}$ & $0.51^{* *}$ & $0.06^{* *}$ & $0.08 *$ & $-2.16^{* *}$ \\
\hline $\begin{array}{c}\text { QPM58 X } \\
\text { QPM-53-2-2 }\end{array}$ & $-25.76^{* *}$ & $1.56^{* *}$ & $1.67 * *$ & 0.10 & $-19.49 * *$ & $-2.49 * *$ & -0.12 & $-0.88^{* *}$ & $-4.55^{* *}$ & $0.96^{*}$ & $-1.07 *$ & $-1.43^{*}$ & $0.55^{* *}$ & $0.08 * *$ & 0.02 & $0.44 * *$ \\
\hline $\begin{array}{l}\text { QPM58 X } \\
\text { QPM-10-1 }\end{array}$ & $-11.76^{* *}$ & $1.05 * *$ & $1.18 * *$ & 0.13 & $-9.92 * *$ & $-1.42 * *$ & $0.58 * *$ & $-1.22 * *$ & $4.94 * *$ & 0.38 & $-2.34 * *$ & 0.42 & $-0.47 *$ & -0.02 & $-0.10^{*}$ & $1.45^{* *}$ \\
\hline $\begin{array}{l}\text { QPM58 X } \\
\text { QPM-45 }\end{array}$ & $9.79 * *$ & $-1.10 * *$ & $-1.27 * *$ & -0.17 & $15.42^{* *}$ & $2.95 * *$ & $0.56^{* *}$ & -0.24 & $-5.79 * *$ & $-2.80 * *$ & $-1.90 * *$ & $2.72 * *$ & $0.89 * *$ & $0.06^{* *}$ & $0.17 * *$ & $0.21^{*}$ \\
\hline $\begin{array}{c}\text { QPM58 X } \\
\text { QPM-15 }\end{array}$ & $14.18^{* *}$ & $-3.50 * *$ & $-3.88 * *$ & -0.38 & $12.90^{* * *}$ & $5.39 * *$ & $1.53^{* *}$ & 0.08 & -0.38 & -0.50 & 0.52 & $8.18 * *$ & $-0.69 * *$ & -0.02 & $0.28 * *$ & $1.24 * *$ \\
\hline $\begin{array}{c}\text { QPM58 X } \\
\text { QPM3-3 }\end{array}$ & $26.12^{* *}$ & $-3.25 * *$ & $-3.21 * *$ & 0.04 & $21.42^{* *}$ & $4.80 * *$ & $1.34 * *$ & 0.38 & $4.73 * *$ & $-1.53^{* *}$ & -0.06 & $3.51 * *$ & $0.38^{*}$ & $0.06 * *$ & $-0.10^{* *}$ & $0.32 * *$ \\
\hline $\begin{array}{l}\text { QPM58 X } \\
\text { QPM-39 }\end{array}$ & -5.82 & $-1.71 * *$ & $-1.67 * *$ & 0.04 & $-2.58 * *$ & $4.73 * *$ & $1.13^{* *}$ & $0.57 *$ & $4.90 * *$ & $-0.95 *$ & $2.80 * *$ & $1.72 * *$ & $-0.41 *$ & $0.04 * *$ & $-0.22 * *$ & $1.01 * *$ \\
\hline $\begin{array}{c}\text { QPM58 X } \\
\text { QPM-77 }\end{array}$ & $19.94 * *$ & $3.41 * *$ & $3.12 * *$ & -0.29 & $20.75^{* *}$ & $4.64 * *$ & $1.11 * *$ & $1.62 * *$ & $4.95 * *$ & $-0.89 *$ & $-1.93 * *$ & -0.61 & -0.28 & -0.02 & $0.24 * *$ & $1.74 * *$ \\
\hline $\begin{array}{c}\text { QPM10057-2 } \\
\text { X QPM-53-2- } \\
2\end{array}$ & $22.67 * *$ & $-4.35 * *$ & $-4.58 * *$ & -0.23 & $12.75^{* *}$ & $0.76^{* *}$ & $1.76^{* *}$ & $1.31^{* *}$ & $6.04 * *$ & $-1.98^{* *}$ & 0.77 & $1.42 *$ & 0.20 & $0.04 * *$ & $-0.17 * *$ & $0.69^{* *}$ \\
\hline $\begin{array}{l}\text { QPM10057-2 } \\
\text { X QPM-10-1 }\end{array}$ & 1.00 & $-1.86^{* *}$ & $-1.73 * *$ & 0.13 & $2.66 * *$ & $5.59^{* *}$ & $2.53 * *$ & $2.00 * *$ & $7.33 * *$ & $-1.22 * *$ & $2.90 * *$ & $5.60 * *$ & $0.51 * *$ & $0.06 * *$ & $-0.10 * *$ & $1.60 * *$ \\
\hline $\begin{array}{c}\text { QPM10057-2 } \\
\text { X QPM-45 }\end{array}$ & $-9.45^{* *}$ & $-1.35^{* *}$ & $-1.52 * *$ & -0.17 & $-2.34 * *$ & $6.13 * *$ & $2.47 * *$ & $1.88^{* *}$ & $8.59 * *$ & $-1.41^{* *}$ & $3.47 * *$ & $4.57 * *$ & $0.88^{* *}$ & $0.04 * *$ & -0.07 & $1.55^{* *}$ \\
\hline $\begin{array}{c}\text { QPM10057-2 } \\
\text { X QPM-15 }\end{array}$ & $-19.39 * *$ & $1.26 * *$ & $1.21 * *$ & -0.05 & $-16.19 * *$ & $-3.90 * *$ & $-1.69 * *$ & -0.33 & $-4.73^{* *}$ & 0.23 & $-2.87 * *$ & $-1.64 * *$ & $-0.61 * *$ & $-0.06^{* *}$ & $0.31^{* *}$ & $0.55^{* *}$ \\
\hline $\begin{array}{c}\text { QPM10057-2 } \\
\text { X QPM3-3 }\end{array}$ & $-14.12 * *$ & $-2.83 * *$ & $-3.12 * *$ & -0.29 & -0.01 & $2.88^{* * *}$ & $-0.78^{* *}$ & $-1.66 * *$ & $-7.85^{* *}$ & $1.20 * *$ & $1.25^{*}$ & 0.36 & $0.47^{*}$ & $0.05 * *$ & $0.21^{* *}$ & $-0.58 * *$ \\
\hline $\begin{array}{c}\text { QPM10057-2 } \\
\text { X QPM-39 }\end{array}$ & $-18.06 * *$ & $-2.95 * *$ & $-2.91 * *$ & 0.04 & 0.99 & $2.57 * *$ & $-0.53 * *$ & $0.76^{* *}$ & $3.55 * *$ & 0.11 & 0.11 & $2.90 * *$ & $0.38^{*}$ & $0.06 * *$ & $0.25 * *$ & $0.96 * *$ \\
\hline $\begin{array}{c}\text { QPM10057-2 } \\
\text { X QPM-77 }\end{array}$ & $8.36^{*}$ & $0.84 * *$ & $0.88^{*}$ & 0.04 & $10.99^{* *}$ & $1.52 * *$ & $-1.01 * *$ & $-1.46 * *$ & $-5.83 * *$ & $-2.50 * *$ & $-4.82 * *$ & $6.57 * *$ & $-1.09 * *$ & $-0.10 * *$ & $0.17 * *$ & $2.20 * *$ \\
\hline $\begin{array}{l}\text { QPM-53-2-2 } \\
\text { X QPM-10-1 }\end{array}$ & $24.97 * *$ & 0.02 & 0.30 & 0.28 & $19.51^{* *}$ & $6.58^{* *}$ & $1.62 * *$ & 0.18 & $-2.66^{* *}$ & $-3.19 * *$ & $3.34 * *$ & $6.66 * *$ & $0.49^{*}$ & 0.02 & $0.16^{* *}$ & $-0.27 * *$ \\
\hline $\begin{array}{c}\text { QPM-53-2-2 } \\
\text { X QPM-45 }\end{array}$ & 4.52 & -0.13 & -0.15 & -0.02 & $-14.82 * *$ & $1.15 * *$ & $-0.78 * *$ & $-0.67 * *$ & $-4.00 * *$ & $2.62 * *$ & $4.04 * *$ & $6.96 * *$ & -0.06 & $-0.04 * *$ & 0.05 & $1.41 * *$ \\
\hline $\begin{array}{c}\text { QPM-53-2-2 } \\
\text { X QPM-15 }\end{array}$ & $8.91 * *$ & $4.81 * *$ & $4.58 * *$ & -0.23 & $-9.34 * *$ & $5.93 * *$ & $1.36^{* *}$ & 0.25 & $-1.65^{*}$ & $0.93^{*}$ & -0.37 & -0.92 & -0.24 & 0.01 & $0.22 * *$ & $0.71 * *$ \\
\hline $\begin{array}{c}\text { QPM-53-2-2 } \\
\text { X QPM3-3 }\end{array}$ & $-26.15 * *$ & $-2.62 * *$ & $-2.42 * *$ & 0.19 & $-15.82 * *$ & $-4.73 * *$ & $-2.36^{* *}$ & 0.32 & -0.28 & $-2.44 * *$ & $1.78 * *$ & 0.08 & 0.13 & $0.03 * *$ & 0.02 & $0.20^{*}$ \\
\hline
\end{tabular}


Table.3 Contd...

\begin{tabular}{|c|c|c|c|c|c|c|c|c|c|c|c|c|c|c|c|c|}
\hline $\begin{array}{c}\text { QPM-53-2-2 } \\
\text { X QPM-39 }\end{array}$ & 1.91 & $-1.41 * *$ & $-1.55 * *$ & -0.14 & $14.84 * *$ & $7.13 * *$ & $3.10 * *$ & $2.31 * *$ & $7.43 * *$ & -0.19 & $1.77 * *$ & $4.63 * *$ & $-0.46 * *$ & $-0.05 * *$ & $0.13 * *$ & $1.22 * *$ \\
\hline $\begin{array}{l}\text { QPM-53-2-2 } \\
\text { X QPM-77 }\end{array}$ & $21.00 * *$ & $-4.95 * *$ & -4.76 ** & 0.19 & $21.18 * *$ & $-2.02 * *$ & -0.22 & -0.14 & -0.15 & $-3.47 * *$ & 0.14 & $4.30^{* *}$ & $0.64 * *$ & 0.00 & $-0.13^{* *}$ & $1.48 * *$ \\
\hline $\begin{array}{c}\text { QPM-10-1 X } \\
\text { QPM-45 }\end{array}$ & $9.85^{* *}$ & $-1.65 * *$ & -1.30 ** & 0.35 & $12.08 * *$ & $-1.71 * *$ & $0.46^{* *}$ & $-0.52 *$ & $-7.07 * *$ & $-2.95 * *$ & 0.07 & $-7.19 * *$ & -0.11 & $-0.09 * *$ & $0.28 * *$ & $0.83 * *$ \\
\hline $\begin{array}{c}\text { QPM-10-1 X } \\
\text { QPM-15 }\end{array}$ & $-14.09 * *$ & $3.62 * *$ & $3.42 * *$ & -0.20 & $-8.10 * *$ & $1.96 * *$ & $-1.24 * *$ & $-0.83 * *$ & $-6.99 * *$ & -0.65 & -0.04 & 0.27 & $0.73 * *$ & $0.09 * *$ & $0.22 * *$ & $0.36 * *$ \\
\hline $\begin{array}{c}\text { QPM-10-1 X } \\
\text { QPM3-3 }\end{array}$ & $15.18^{* *}$ & $-1.47 * *$ & $-1.58 * *$ & -0.11 & $4.75 * *$ & $6.44 * *$ & $2.84 * *$ & $2.84 * *$ & $10.05^{* *}$ & $-2.35 * *$ & $2.98 * *$ & $6.93 * *$ & $1.07 * *$ & $0.08 * *$ & 0.06 & $1.92 * *$ \\
\hline $\begin{array}{c}\text { QPM-10-1 X } \\
\text { QPM-39 }\end{array}$ & $9.91 * *$ & $-2.59 * *$ & $-2.70 * *$ & -0.11 & $2.75 * *$ & $-5.80 * *$ & $-1.70 * *$ & $-1.41 * *$ & $-3.68 * *$ & $0.90^{*}$ & $-1.73 * *$ & $3.48 * *$ & -0.25 & $-0.05^{* *}$ & $0.23 * *$ & $0.55 * *$ \\
\hline $\begin{array}{c}\text { QPM-10-1 X } \\
\text { QPM-77 }\end{array}$ & $-23.67 * *$ & $-6.13 * *$ & $-5.91 * *$ & 0.22 & $-19.25 * *$ & $-4.15 * *$ & $-0.79 * *$ & $-0.56^{*}$ & $-1.59 *$ & $-1.38 * *$ & $1.08^{*}$ & 0.15 & $1.15^{* *}$ & $0.04 * *$ & $-0.16^{* *}$ & $-0.80 * *$ \\
\hline $\begin{array}{l}\text { QPM-45 X } \\
\text { QPM-15 }\end{array}$ & $-13.88 * *$ & $-2.53 * *$ & $-2.36 * *$ & 0.16 & -1.43 & $8.73 * *$ & $3.67 * *$ & $2.59 * *$ & $17.41 * *$ & $-0.83^{*}$ & $6.60 * *$ & $6.57 * *$ & $0.93 * *$ & $0.07 * *$ & 0.04 & $1.90 * *$ \\
\hline $\begin{array}{c}\text { QPM-45 X } \\
\text { QPM3-3 }\end{array}$ & $-7.61 *$ & $-2.28 * *$ & $-2.36^{* *}$ & -0.08 & -0.92 & $-6.56^{* *}$ & $-1.35^{* *}$ & $-1.27 * *$ & $-6.48 * *$ & -0.19 & $-3.82 * *$ & $4.57 * *$ & $-0.33^{*}$ & $-0.05^{* *}$ & $0.17 * *$ & $1.09 * *$ \\
\hline $\begin{array}{c}\text { QPM-45 X } \\
\text { QPM-39 }\end{array}$ & $-13.55 * *$ & $0.93 * *$ & $0.85^{*}$ & -0.08 & $-11.25 * *$ & $6.17 * *$ & $2.34 * *$ & $2.24 * *$ & $9.98 * *$ & $-1.28 * *$ & $3.17 * *$ & $3.78 * *$ & $1.21 * *$ & $0.08 * *$ & $-0.18 * *$ & $1.10 * *$ \\
\hline $\begin{array}{c}\text { QPM-45 X } \\
\text { QPM-77 }\end{array}$ & -1.79 & $-0.95 * *$ & $-1.03 * *$ & -0.08 & $3.75 * *$ & 0.42 & $2.12 * *$ & $1.83 * *$ & $6.64 * *$ & $1.11 * *$ & $1.75^{* *}$ & $3.12 * *$ & $0.92 * *$ & 0.01 & $-0.30 * *$ & $0.75 * *$ \\
\hline $\begin{array}{l}\text { QPM-15 X } \\
\text { QPM3-3 }\end{array}$ & $-8.55^{*}$ & $-0.68^{*}$ & -0.64 & 0.04 & $-8.43 * *$ & $-5.29 * *$ & $-1.91 * *$ & $-1.92 * *$ & $-2.54 * *$ & $-1.89 * *$ & 0.81 & $-1.98 * *$ & $-0.51 * *$ & -0.01 & $0.10^{* *}$ & $1.64 * *$ \\
\hline $\begin{array}{l}\text { QPM-15 X } \\
\text { QPM-39 }\end{array}$ & -2.82 & $-4.13 * *$ & $-4.42 * *$ & -0.29 & $7.57 * *$ & $-6.46^{* *}$ & $-2.33 * *$ & $-2.24 * *$ & $-6.10 * *$ & $1.35 * *$ & $-2.23 * *$ & $-2.43^{* *}$ & -0.17 & $0.06 * *$ & -0.04 & $0.43 * *$ \\
\hline $\begin{array}{c}\text { QPM-15 X } \\
\text { QPM-77 }\end{array}$ & $-15.39 * *$ & $-4.35 * *$ & $-4.64 * *$ & -0.29 & $-13.10 * *$ & $-3.24 * *$ & $0.73 * *$ & -0.32 & $-3.25 * *$ & $-2.25 * *$ & $1.87 * *$ & $1.90 * *$ & $0.83 * *$ & $0.07 * *$ & 0.05 & $-1.98 * *$ \\
\hline $\begin{array}{c}\text { QPM3-3 X } \\
\text { QPM-39 }\end{array}$ & $-26.88 * *$ & $-2.89 * *$ & $-2.09 * *$ & $0.80 * *$ & $-15.25 * *$ & $5.85 * *$ & $1.59 * *$ & $-1.10 * *$ & $-10.09 * *$ & $2.32 * *$ & $-1.22 *$ & $-1.43^{*}$ & $0.27 *$ & -0.01 & $0.12 * *$ & $0.75 * *$ \\
\hline $\begin{array}{c}\text { QPM3-3 X } \\
\text { QPM-77 }\end{array}$ & $19.21 * *$ & $-3.10^{* *}$ & $-3.30 * *$ & -0.20 & $5.42 * *$ & $6.97 * *$ & $2.57 * *$ & $2.05 * *$ & $7.26^{* *}$ & $1.38 * *$ & 0.36 & -0.10 & $1.44 * *$ & $0.07 * *$ & -0.10 ** & $-1.27 * *$ \\
\hline $\begin{array}{c}\text { QPM-39 X } \\
\text { QPM-77 }\end{array}$ & $-18.39 * *$ & $-2.56^{* *}$ & $-2.42 * *$ & 0.13 & $-11.92 * *$ & $-3.01 * *$ & $-1.47 * *$ & $-1.03 * *$ & -1.20 & $-1.38 * *$ & 0.51 & $1.78 * *$ & 0.18 & $-0.03^{*}$ & 0.05 & $1.01 * *$ \\
\hline SE & 3.224 & 0.283 & 0.343 & 0.210 & 0.665 & 0.212 & 0.144 & 0.212 & 0.643 & 0.321 & 0.516 & 0.576 & 0.131 & 0.010 & 0.035 & 0.88 \\
\hline Sij-Sik & 4.754 & 0.418 & 0.505 & 0.351 & 1.109 & 0.312 & 0.240 & 0.313 & 0.949 & 0.535 & 0.760 & 0.849 & 0.193 & 0.015 & 0.051 & 1.30 \\
\hline Sij-Skl & 4.510 & 0.396 & 0.480 & 0.333 & 1.052 & 0.296 & 0.228 & 0.297 & 0.900 & 0.507 & 0.721 & 0.806 & 0.183 & 0.015 & 0.048 & 1.23 \\
\hline
\end{tabular}

* Significant at $5 \%$ level; ** Significant at $1 \%$ level 
Three parents viz., QPM58, QPM-15 and QPM-77 which expressed significant negative gca effects were found as good general combiners for reduced plant height. These results are in agreement with the findings of Bhavana et al., (2011) and Sumalini (2012) who reported the additive gene action for plant height.

The best three specific crosses for ear height were, QPM-58 X QPM-3-3, QPM58 X QPM77 and QPM-53-2-2 X QPM-77 with the highest significantly positive sca effects.

Four parents viz., QPM10057-2, QPM-45, QPM-3-3 and QPM-39 exhibited significantly positive $\mathrm{gca}$ effects and thus identified as good combiners for the production of genotypes with larger ear girth. These results are comparable with the findings of Bhavana et al., (2011) and Sumalini (2012) who reported the additive gene action for ear girth. The best three specific crosses for ear girth were QPM-45 X QPM-15, QPM-53-2-2 X QPM-77 and QPM-10-1 X QPM 3-3. Diversion of more assimilates towards the developing kernels will also be responsible for development of cobs with more ear girth which may result in superior hybrids when coupled with higher kernel density. These results are in concurrence with the findings of Jaya Kumar et al., (2007), Ram Reddy et al., (2012) and Sunil Kumar et al., (2012) who reported the non-additive gene action for ear girth.

The parental lines, QPM10057-2, QPM58, QPM-39, QPM-45, QPM-10-1 and QPM-3-3 and recorded significantly positive $\mathrm{gca}$ effects indicating that they were good combiners for ear length. The best specific crosses for ear length were QPM-45 X QPM-15, QPM-53-22 X QPM-39 and QPM-3-3 x QPM-77 which indicated the role of good combiners in developing the superior hybrids for the trait. These results are comparable with findings of
Sumalini and Shobha Rani (2011) and Kumar et al., (2012) who reported the non-additive gene action for ear length.

Five parents viz., QPM10057-2, QPM-45, QPM-15, QPM-3-3 and QPM-39 recorded significantly positive gca effects for Number of Kernel rows per ear. The best specific crosses for number of kernel rows per ear were QPM-10-1 X QPM-3-3, QPM-45 X QPM-15 and QPM-53-2-2 X QPM-39. It is evident from the above that parental lines with high gca effects resulted in superior hybrids for the trait. These results are comparable with findings of Sumalini et al., (2012) and Sunil Kumar et al., (2012) who reported the non-additive gene action for number of kernel rows per ear.

Among the parents, QPM58, QPM10057-2, QPM-45 and QPM-39 exhibited significantly superior positive gca effects for Number of kernels per row. Among the tested hybrids, QPM 45 X QPM-15, QPM - 10-1 X QPM 33 and QPM-45 X QPM-39 identified as the best specific combiners with the superior positive sca effects which also indicated the role of parental lines with high gca effects in the production of superior hybrids for the trait. These results are comparable with findings of Pavan et al., (2011), Ram Reddy et al., (2011) and Sunil Kumar et al., (2012) who reported the non-additive gene action for number of kernels per row.

The parents, QPM10057-2, QPM-45, QPM15, QPM-3-3 and QPM-39 exhibited significantly positive gca effects for 100kernel weight. The hybrids, QPM-45 x QPM15 and QPM 10057-2 X QPM-45 were identified as best ones based on their superior positive sca effects which indicated the predominant role of parental lines with high gca effects in production of superior hybrids. The parents, QPM10057-2, QPM-45 and QPM-39 exhibited significantly positive gca 
effects and identified as good combiners for the trait.). These results are comparable with findings of Jaya Kumar et al., (2007) and Ankit Kumar et al., (2017) who reported the additive gene action for shelling percentage. The hybrids, QPM-58 x QPM-15 and QPM 53-2-2 X QPM-45 were identified as best ones based on their superior positive sca effects. These results are comparable with findings of Jaya Kumar et al., (2007) who reported the non-additive gene action for shelling percentage.

Significantly positive gca effects were observed in four parents viz., QPM58, QPM10057-2, QPM 53-2-2 and QPM-15 were identified as good combiners for crude protein content. These results are comparable with findings of Machida et al., (2010), Firoz Husain (2007), Shanti et al., (2011) and Amiruzzaman et al., (2012) who reported the additive gene action for crude protein content. The hybrids, QPM-3-3 x QPM-77, QPM 45 X QPM-39 and QPM 10-1 X QPM-77 recorded superior positive sca effects and thus identified as best hybrids. These results are in accordance with the earlier findings of Mohan lal (2011), Amiruzzaman et al., (2012) who reported non additive gene action for crude protein content.

Three parents viz. QPM10057-2, QPM 45 and QPM-77 exhibited significantly positive $\mathrm{gca}$ effects for tryptophan content. The hybrids, QPM 10-1 X QPM-15, QPM 10-1 X QPM 33 and QPM-45 X QPM-39 were identified as best hybrids based on their superior positive sca effects. These results are in accordance with the earlier findings of Mohan lal (2011), Amiruzzaman et al., (2012) who reported non additive gene action for tryptophan content.

Three parents viz., QPM10057-2, QPM45 and QPM-15 exhibited significantly positive gca effects and identified as good combiners for lysine content. These results are comparable with the findings of Amiruzzaman et al., (2012) who reported the additive gene action. The hybrids, QPM10057-2 X QPM-15, QPM 10-1 X QPM-45, QPM -58 X QPM-15, QPM10057-2 X QPM-39 were identified as best hybrids based on their superior positive sca effects indicating the predominant role of parental lines with high $\mathrm{gca}$ in producing the superior hybrids for the trait. These results are in accordance with the earlier findings of Mohan lal (2011), Amiruzzaman et al., (2012) who reported non additive gene action for lysine content.

Five parents viz., QPM 10057-2, QPM-10-1, QPM-45, QPM-15 and QPM-39 exhibited significantly positive effects and thus identified as good general combiners. Hence, these parents will be utilized in the development of hybrids, synthetics and composites which are in accordance with the findings of Mohammad (1993) and Mathur et al., (1998) who reported the additive gene action for grain yield. The hybrids, QPM10057-2 X QPM-77, QPM 10-1 X QPM-3-3, QPM-45 X QPM-1539 were identified as best hybrids based on their superior positive $s c a$ effects.

The parents, QPM-39, QPM-45, QPM $10057-$ 2, QPM-10-1 and QPM-15 can be considered as good general combiners and genetically worthy parents, as they contributed favourable genes for grain yield and its components at all the three locations and in pooled analysis.

Combining ability analysis revealed that the parents, QPM58, QPM-45, QPM-3-3, QPM39 and QPM-15 were found to be good general combiners for earliness. The parent, QPM-39 and QPM-45 were good general combiners for grain yield, days to 50 per cent anthesis, days to 50 per cent silking, days to maturity (all towards earliness), ear girth, ear length, number of kernels per ear, number of 
kernels per row, 100-kernel weight, shelling percentage. In addition, QPM-45 was a good combiner for tryptophan and lysine contents.

\section{References}

Amiruzzaman, M., Islam, M. A., Pixley, K. V and Rohman, M.M 2012. Heterosis and combining ability of CIMMYT's Tropical $\times$ Subtropical Quality Protein Maize germplasm. International Journal of Sustainable Agriculture. 3(3): 76-81.

Ankit Kumar, R. P., Vyas., Amit Tomar and Mahak Singh. 2017. Genetic components analysis in maize (Zea mays L.). The Pharma Innovation Journal. 6(8): 315-317

Bhavana, P., Singh, R. P and Gadag, R. N. 2011. Gene action and heterosis for yield and yield components in maize (Zea mays L.). Indian Journal of Agricultural Sciences. 81 (2): 163166.

Firoz Hossain., Tripati, S and Warsi, M. Z. K. 2007. Inter character correlation and coefficient of variation in inbreds and their hybrids of maize grown under normal and water logged condition. Biulyn instytutu Holowli-iAklimatyazacji Roslin. 23 (1): 419424.

Jagadish Kumar., Sanjeev Kumar and Sood, B.C. 2010. Gene action for yield and yield contributing characters in indigenous maize (Zea mays L.) germplasm of Himachal Pradesh. Crop Improvement. 37 (2): 114-19.

Jayakumar, J., Sundaram, T., Prabu, D. A and Rajan, A. R. R. 2007. Correlation studies in maize evaluated for grain yield and other yield attributes. International Journal of Agricultural Sciences. 3: 57-60.

Lata, S., Sharma, J. K., Katna and Guleria. 2008. Heterosis and combining ability for polygenic traits in medium maturity hybrids of maize (Zea mays L.). Crop Improvement. 35 (2): 135138.

Machida L, Derera J, Tongoona P, MacRobert J. 2010. Combining ability and reciprocal cross effects of elite quality protein maize inbred lines in subtropical environments. Crop Science. 50: 1708- 1717.

Mathur, R. K., Chunilal, Bhatnagar, S. K and Singh, V. 1998. Combining ability for grain yield, phonological and ear characters in white seeded maize. (Zea mays L.). Indian Journal of Genics and Plant Breeding. 58 (2): 117-182.

Mohammed, A. A. 1993. Effect of nitrogen fertilization levels on performance and combining ability of maize (Zea mays L.). Annals of Agricultural Science. 38 (2): 531-549.

Mohan Lal, Dhirendra Singh and Sain Dass. 2011. General and specific combining ability studies in maize using line $\mathrm{x}$ tester design. Agricultural Science Digest. 31 (1): $8-13$

Murthy, A. R., Kajjari, N. B and Goud, J. V. 1981. Diallel analysis of yield and maturity components in maize. Indian Journal of Genics and Plant Breeding. 41: 30-33.

Kumar, B. S., Prakash, M., Sathyanarayanan, G. and Padmavathi, S. 2012. Studies on combining ability and heterosis through line $\mathrm{x}$ tester analysis in maize (Zea mays L.). Crop Research (Hisar). 43 (1/2/3): 153-157.

Pavan, R., Lohithaswa, H. C., Wali, M. C., Gangashetty Prakash and Shekara, B.G. 2011. Correlation and path analysis of grain yield and yield contributing traits in single cross hybrids of maize (Zea mays L.). Electronic Journal of Plant Breeding. 2 (2): 253-257.

Ram Reddy, V., Seshagiri Rao, A and 
Sudarshan, M. R. 2012. Heritability and character association among grain yield and its components in maize (Zea mays L.). Journal of Research, ANGRAU. 40(2): 45-49.

Sanghi, A. K., Agarwal, K. N and Qadri, M. I. 1982. Gene effects and heterosis for grain yield and ear traits in maize. Indian Journal of Genics and Plant Breeding. 42: 360-363.

Shanthi, P., Babu, G. S., Satyanarayana, E and Kumar, R.S. 2011. Combining ability and stability studies for grain yield and quality parameters in QPM (Zea mays L.) inbred line crosses. Indian Journal of Genics. 70(1): 2229.

Singh, S. B., Gupta, B. B and Anjani K. S. 2010. Heterotic expression and combining ability for yield and its components in maize (Zea mays L.).
Progressive Agriculture. 10 (2): 275281.

Sumalini, K and Shobha Rani, T. 2011. Heterosis and combining ability for polygenic traits in late maturity hybrids of maize (Zea mays L.). Madras Agricultural Journal. 97 (1012): 340-343.

Sumalini, K. 2012. Combining ability and heterosis for yield and quantitative traits in maize (Zea mays L.). Madras Agricultural Journal. 99 (4-6): 188191.

Sunil Kumar, B., Prakash, M., Sathyanarayanan, G. and Padmavathi, S. 2012. Studies on combining ability and hetrosis through line $\times$ tester analysis in maize (Zea mays L.). Crop Research, Hisar. 43 (1, 2 \& 3): 153157.

\section{How to cite this article:}

Sukumar, K., V. Hemalatha, V. Narsimha Reddy, S. Narender Reddy and Srinivasa Chary, D. 2020. Combining Ability Studies in Quality Protein Maize (Zea mays L.). Int.J.Curr.Microbiol.App.Sci. 9(07): 4123-4134. doi: https://doi.org/10.20546/ijcmas.2020.907.485 